Diagnostic Radiology and Nuclear Medicine. Yonsei University College of Medicine, Seoul, 120-752, Korea. E-mail: jdlee'i yuhs.ac).

COMMENT. Pathophysiological mechanisms of motor impairment in patients with periventricular leukomalacia and spastic cerebral palsy are controversial (Bax et al, JAMA 2006; Woodward et al, N Engl J Med 2006) The above study demonstrates that descending motor pathway injury and reduction of overlying cortical volume are leading mechanisms.

Neuronal cell death in neonatal hypoxia-ischemia, reviewed by researchers at Johns Hopkins University School of Medicine (Northington FJ et al. Ann Neurol May 2011;69:743-758), is manifested as a continuum of programmed cell death (PCD) from apoptosis (dismantling of cells, Type I PCD) to necrosis (lytic destruction of cells) and autophagy (degradation of cells by lysosomal system, Type II PCD), and not as distinct categories of neurocellular degeneration. Hypothermia in infants with HIE protects against necrosis and apoptosis.

\title{
NEURODEVELOPMENTAL DISORDERS
}

\section{NEUROANATOMICAL ABNORMALITIES IN BOYS WITH IDIOPATHIC AUTISM COMPARED TO FRAGILE X SYNDROME}

Researchers at Stanford University, CA, and University of North Carolina, Chapel Hill, used voxel-based morphometric analyses, and multivariate pattern and clustering analyses to compare brain MRI studies in 165 young boys, aged 1.57- 4.15 years, diagnosed with idiopathic autism (iAUT) or fragile X syndrome (FXS) and idiopathic developmentally delayed and normal controls. Frontal and temporal gray and white matter regions of brains of boys with iAUT and FXS were developmentally aberrant as compared with controls. The differences were in opposite directions in iAUT and FXS relative to controls; iAUT brains had greater volume compared to controls, and FXS brains had smaller volume than control brains. Pattern of brain structure in iAUT generally resembled that of controls more than FXS, with or without autism. (Hoeft F, Waiter E, Lightbody AA et al. Neuroanatomical differences in toddler boys with fragile X syndrome and idiopathic autism. Arch Gen Psychiatry March 2011;68(3):295-305). (Response: Allan L Reiss MD, Dept Psychiatry and Behavioral Sciences, Stanford University School of Medicine, Stanford, CA 94305. E-mail: reiss@stanford.edu).

COMMENT. The authors conclude that idiopathic autism and fragile $\mathrm{X}$ syndrome have distinct neuroanatomical patterns, a finding that supports the neurobiological heterogeneity of idiopathic autism. They suggest that significant differences in aberrant brain morphometry may be found in other ASD-associated genetic disorders, eg Angelman syndrome, Rett syndrome. ASD has been diagnosed in more than 20 genetically determined syndromes, most prominently, FXS. (Harris JC. Editorial 2011; Moss J et al. 2009). 
Brain size in autism. Cerebral cortical enlargement is reported in children with ASD examined by MRI at both 2 and 4-5 years of age (Hazlett HC et al. Arch Gen Psychiatry May 2011;68(5):467-476). A disproportionate enlargement in temporal lobe white matter was observed in ASD children $(\mathrm{n}=38) \mathrm{cf}$ controls $(\mathrm{n}=21)$. No significant difference from controls was observed in the rate of brain growth between 2 and 4 years of age. Brain enlargement observed in children with ASD is a consequence of an increased rate of brain growth with onset postnatally, before age 2 years and probably in the latter part of the first year of life. This early brain enlargement with increased cortical volume, but not cortical thickness, may be associated with increased cortical surface area and a distinct pathogenic mechanism. The early cortical overgrowth in young children with ASD is followed by a period of cortical thinning during adolescence and adulthood. (Wallace GL et al. Brain 2010;133:3745-3754).

\section{ARACHNOID CYSTS CAUSING SYMPTOMATIC INCREASED INTRACRANIAL PRESSURE}

Four children, ages 12 to 15 years, with intracranial arachnoid cysts complicated by increased intracranial pressure are reported from Children's Hospital of Philadelphia, PA. They presented with recent onset or increasing headaches, exacerbated by the supine position. Visual acuity was normal. Optic discs were swollen. Opening pressures at LP were 37 to $54 \mathrm{~cm} \mathrm{H} 2 \mathrm{O}$. All failed to respond to conservative medical therapies, but symptoms improved after a surgical shunting procedure. The arachnoid cyst was in the left middle temporal fossa and was complicated by a Chiari I malformation in 3 patients; one patient had hydrocephalus diagnosed at 18 months and a posterior fossa cyst. Three patients were previously treated with a cystoperitoneal shunt before age 2 and required shunt revision. (Prasad S, Avery RA, Campomanes A, Sutton LN, Liu GT. Symptomatic increased intracranial pressure due to arachnoid cysts. Pediatr Neurol May 2011;44:377380). (Respond: Dr Prasad, Division of Neuro-Ophthalmology, Brigham and Women's Hospital, 75 Francis St, Boston, MA 02115. E-mail: sprasad2@partners.org).

COMMENT. Usually benign, arachnoid cysts are rarely complicated by increased intracranial pressure that may require a surgical shunt procedure. The association of a Chiari I malformation with an arachnoid cyst in 3 of these patients is noteworthy, but a possible relation to the headaches and increased intracranial pressure is undetermined. Other symptoms sometimes associated with arachnoid cyst include attention deficit hyperactivity disorder, not alluded to in this report.

\section{OUTCOMES OF BILATERAL STURGE-WEBER SYNDROME}

Researchers at Wayne State University School of Medicine, Detroit, MI studied 14 children with bilateral Sturge-Weber syndrome, representing $12.7 \%$ of a total of 110 patients with the disorder. Glucose positron emission tomography had an asymmetric pattern, one hemisphere was hypometabolic in 3 patients. Signs of cerebral atrophy or vascular abnormalities on MRI were more pronounced on one side. Long-term follow-up data available for 8 of the 14 patients covered 1-16 years (median, 9 years). Age at onset of seizures in 14 patients ranged from 2 weeks to 7.1 years (median, 6 months). Seizure 